\title{
Diversity Method in the Chaos CDSK Communication System
}

\author{
Jun-Hyun Lee and Heung-Gyoon Ryu \\ Department of Electronic Engineering, Chungbuk National University, Korea \\ toogee89@nate.com, ecomm@cbu.ac.kr
}

\begin{abstract}
Chaos communication system has characteristics of non-periodic, non-predictability, broadband signal and easy implementation. Also, the characteristic of chaos communication system is decided by initial values. And it has the sensitive characteristic according to initial values. Due to these characteristics, security of chaos communication system is generally better than other digital communication system. But BER(Bit Error Rate) performance is worse than digital communication system. Looking at the existing studies, chaos communication system is being studied diversity method in order to improve the BER performance in Rayleigh channel. In this paper, we apply the Alamouti STBC(Alamouti Space Time Block Coding) in order to improve the BER performance, and we evaluate BER performance of CDSK system that applied the Alamouti STBC. If we apply the Alamouti STBC to the CDSK system, we are possible to evaluate the BER performance improvement in Rayleigh channel. Therefore, if the Alamouti STBC of MIMO(Multi Input Multi Output) system applies to chaos communication system in fading channel, the deterioration of BER performance is possible to compensate. And, if you adjust number of transmitter and receiver according to kinds of channels, effective communication is possible.
\end{abstract}

Keywords - Correlation Delay Shift Keying; MIMO; Alamouti STBC; BER performance; Chaos Map.

\section{INTRODUCTION}

Previous digital communication technology used continually a linear system. However, this technology reached basic limit, and people study nonlinear communication systems that apply chaos communication system in order to improve the performance[1]. Chaos signal has characteristics such as the non-periodicity, broadband signal, nonpredictability and easy implementation. And, chaos communication system is decided by initial values of the equation that generated from chaos signal generator. And it has the sensitive characteristic according to initial values. Because chaos signal is changed to completely different signal according to changing initial values or parameters[2-3]. Chaos signal has characteristics such as nonlinear and random signal. And it is possible to predict in principle and determine the value of the future by the value of the past. However, chaos signal has a sensitive characteristic to initial values. If users of chaos communication system do not know the exact initial values, it is impossible to predict the value of the future[1][4].

By combining characteristics of chaos signal and digital communication technologies, this study makes that difficult to detect other user's signal, interfere with waves, and have strong features in the jamming. And it is possible to reduce the probability of eavesdropping. Namely, chaos communication system has the high security more than other systems. But, chaos communication system has the BER performance deterioration larger than digital communication system, and BER performance of chaos communication system is evaluated very badly in fading channel[4]. Therefore, we must find ways in order to improve the BER performance of the chaos communication system in fading channel. By the deterioration of BER performance, existing chaos communication system evaluates the BER performance of several chaos maps, and user of chaos communication system uses selectively chaos map that has the best BER performance. Also, the existing study of chaos communication system evaluate the BER performance according to kind of chaos modulation system, and security[5]. In addition, studies of chaos communication system are performed continuously in order to improve the BER performance by applying the MIMO system in fading channel. And, chaos communication system that is applied MIMO system is studied in various channel[6-7].

In a mobile communication environment, multipath fading is a disorder greatest factor for realizing high-speed data communication. However, if chaos communication system is applied the MIMO system, performance of system is possible to improve[8]. Therefore, BER performance that is deteriorated due to fading channel environment can be improved by applying the MIMO system. Also, BER performance deterioration of the chaos communication system in multi-path fading channel can be solved by applying the MIMO system. It is possible to obtain diversity gain and array gain. These gains have a good effect on the BER performance[9].

In this paper, in CDSK(Correlation Delay Shift Keying) system, we apply the Alamouti STBC(Alamouti Space Time Block Coding) that uses two transmitter and one receiver in order to improve the BER performance at Rayleigh channel. And, we apply the Alamouti STBC that uses two transmitters and two receivers.

\section{SYSTEM OVERVIEW}

Modulation scheme of chaos communication system is used in this paper. 


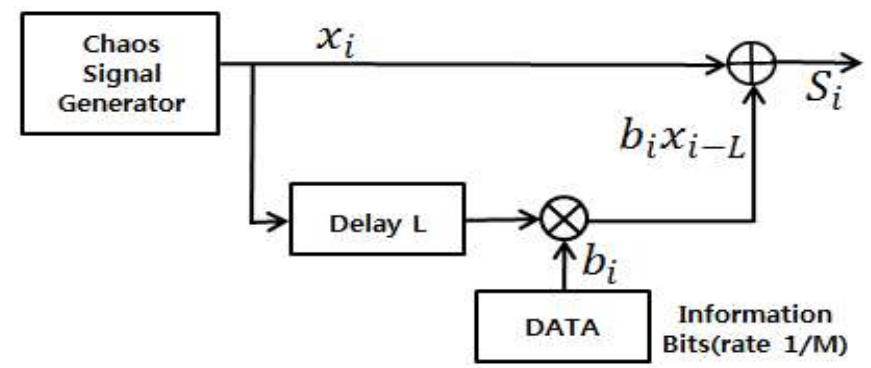

Figure 1. CDSK transmitter system.

CDSK system is one of the modulation systems based on chaos to solve a problem that a transmitted signal does not repeat by replacing an adder with a switch in the transmitter of existing modulation system[10]. If this problem does not solve, the system has problems of eavesdropping and wasted power.

$$
S_{i}=x_{i}+b_{i} x_{i-L}
$$

Figure 1 represents a transmitter of CDSK system. The transmitted signal of the CDSK system is the sum of a chaos signal that is generated from chaos signal generator and a delay chaos signal that is modulated information bits. And a transmitted signal $\left(s_{i}\right)$ is expressed as the equation (1).

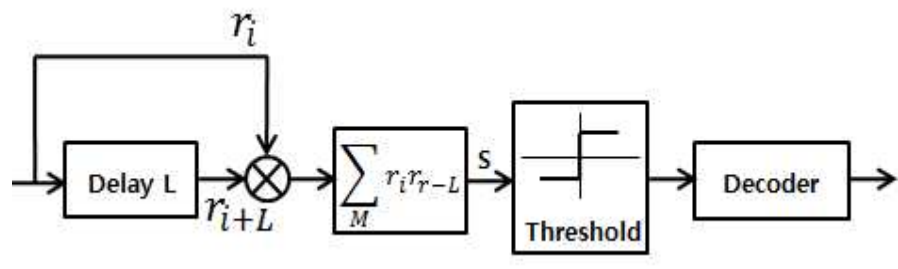

Figure 2. CDSK Receiver system.

Figure 2 represents a receiver of CDSK system. CDSK receiver has a characteristic that the spreading factor and the delay time are not same.

$$
S=\sum_{i=1}^{M} r_{i} r_{i-L}
$$

Equation (2) shows a receiver of CDSK, L means delayed time, and $\mathrm{M}$ points to a spreading factor.

Tent Map of the chaos map is used in this paper. The equation of tent map uses the value of previous output by value of the current input.

$$
x_{n+1}=\left\{\begin{array}{cc}
\alpha x_{n} & \text { for } x_{n}<0.5 \\
\alpha\left(1-x_{n}\right) & \text { for } 0.5 \leq x_{n}
\end{array}\right.
$$

Equation of a Tent map is expressed as equation (3) and draws a trajectory as shown in the figure 3 when you set value of $\alpha$ with 1.99999. Looking at the figure 3, $\mathrm{x}$-axis and $\mathrm{y}$-axis means each $x_{n}, x_{n+1}$.

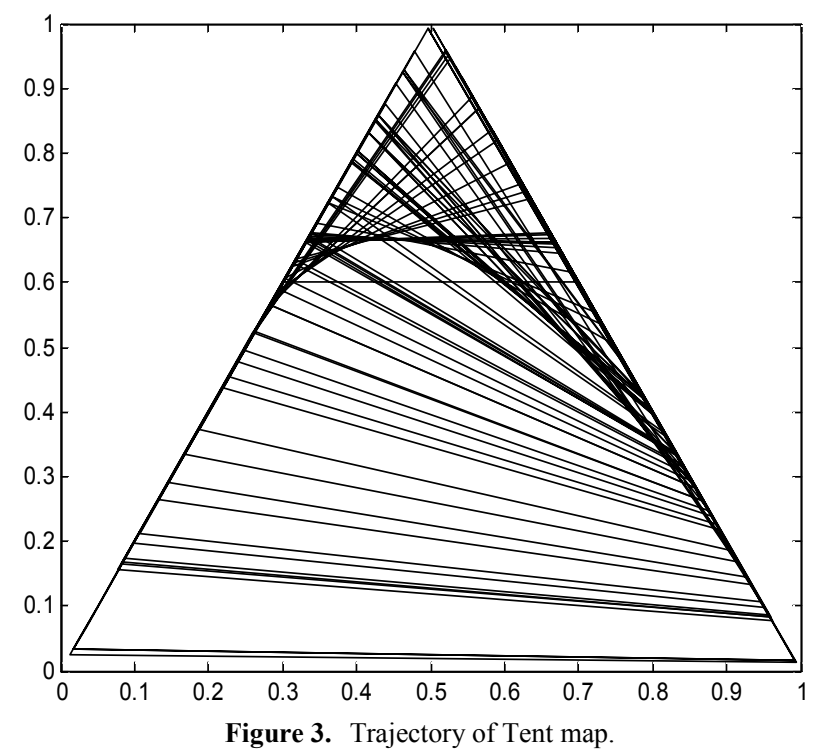

MIMO system is a smart antenna technology in order to increase the capacity of the wireless communication. MIMO system is possible to increase the capacity in proportion to the number of antennas by using a plurality of antennas. Further, in the fading channel, if transmitters and receivers are used, system is possible to improve performance by using effectively the diversity.

The Alamouti STBC of MIMO system is used in this paper. Alamouti STBC suggests that it group symbols into groups of two. The first time slot send $\mathrm{x} 1$ and $\mathrm{x} 2$ from the first and second antenna. And, second time slot send $-\mathrm{x} 2 *$ and $\mathrm{x} 1$ from the first and second antenna. Although it is grouping two symbols, it still needs two time slots to send two symbols. Thus, there is no change in the data rate [5][9].

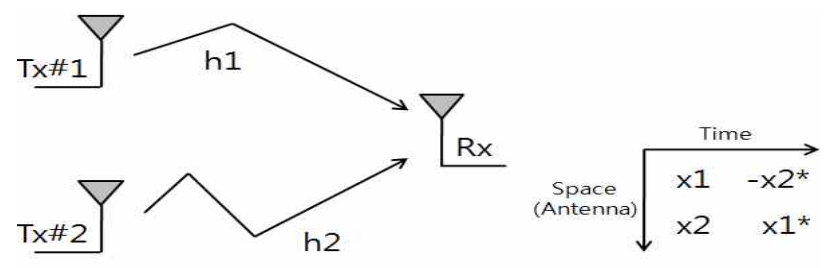

Figure 4. 2-Transmit, 1-Receive Alamouti STBC.

If symbols is transmitted such as Figure 4, characteristic of the channel has orthogonality. If it has orthogonality, you can be easily separated characteristics of the channel mathematically.

$$
\begin{gathered}
y_{1}=h_{1} x_{1}+h_{2} x_{2}+n_{1} \\
y_{2}=-h_{1} x_{2}^{*}+h_{2} x_{1}^{*}+n_{2}
\end{gathered}
$$




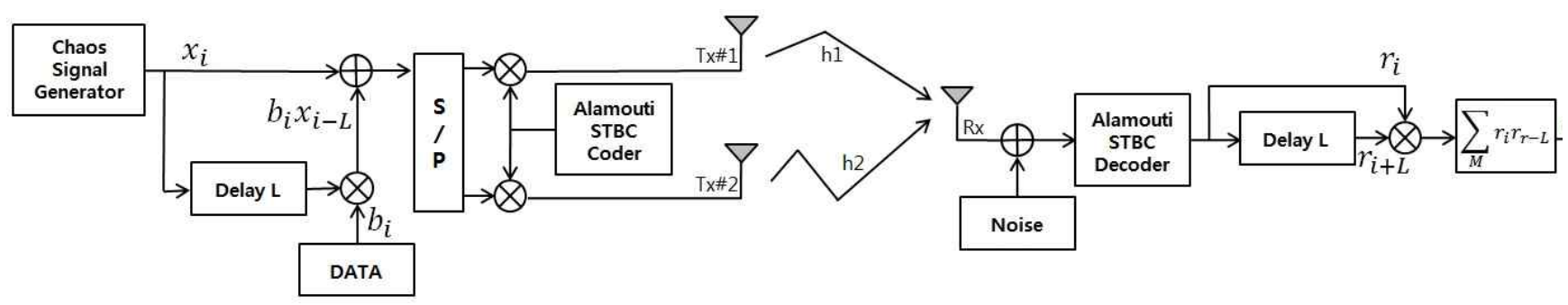

Figure 5. 2-Transmit, 1-Receive Alamouti STBC.

In the first time slot, the received signal is expressed as equation (4). And, in the second time slot, the received signal is expressed as equation (5).

$y_{1}, y_{2}$ are received symbols on the first and second time slot respectively. $h_{1}$ is the channel from first transmitter antenna to receiver antenna, $h_{2}$ is the channel from second transmitter antenna to receiver antenna. $x_{1}, x_{2}$ are the transmitted symbols and $n_{1}, n_{2}$ are the noise on first and second time slots.

Figure 5 shows a block diagram of CDSK system applying the Alamouti STBC of MIMO system. Looking at the figure 5, the transmitter has Alamouti STBC Coder and the receiver has Alamouti STBC Decoder. Through this process, it is possible to improve the BER performance in Rayleigh channels.

\section{III.SIMULATION RESULT}

Flat Rayleigh channel is used in this paper. In simple terms, its mean that the multipath channel has only one tap. So, the convoluted operation reduces to a simple multiplication.

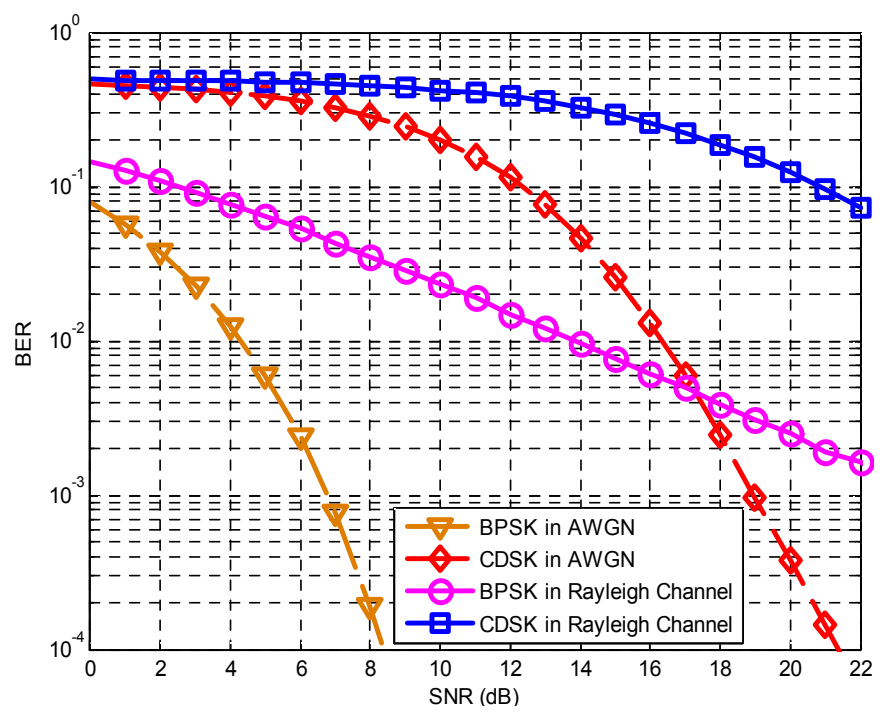

Figure 6. 2-Transmit, 1-Receive Alamouti STBC.।

Figure 6 represents the BER performance of CDSK system in Rayleigh channel. Looking at the figure 6, BER performance of CDSK system is represented very badly in Rayleigh channel.

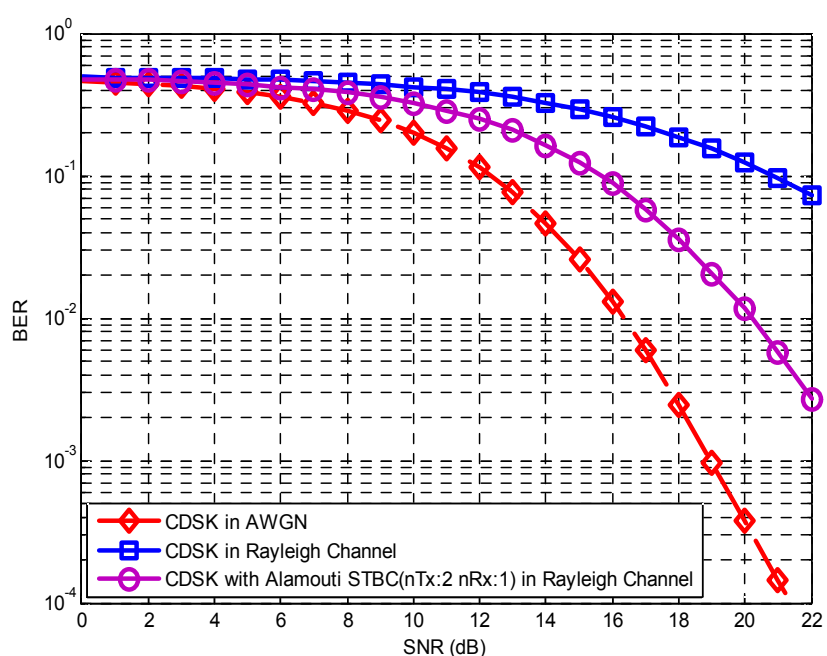

Figure 7. BER performance of CDSK system using Alamouti STBC(nTx:2, nRx:1).।

Figure 7 represents the BER performance of CDSK system that is applied the Alamouti STBC using two transmitters and one receiver. Looking at the figure 7 , the BER performance deterioration can be compensated in Rayleigh channel by applying the Alamouti STBC using two transmitters and one receiver. If CDSK system is applied the Alamouti STBC using the two transmit antenna, it is possible to obtain space-time transmitted diversity[10]. So, BER performance is better than CDSK system without Alamouti STBC.



Figure 8. BER performance of CDSK system using Alamouti STBC(nTx:2, nRx:2). 
Figure 8 represents the BER performance of CDSK system that is applied the Alamouti STBC using the two transmitters and two receiver. Looking at the figure 8 , the BER performance deterioration can be compensated in Rayleigh channel by applying the Alamouti STBC using the two transmitters and two receivers. This BER performance is similar BER performance of AWGN environment. If a method that receiver antennas set spatially far away and two equal symbols is received is selected, it is possible to obtain array gain. And, if systems are obtained the array gain, BER performance is better than previous performance. Example, if two receivers are received 1 and -1 , these symbols are added as 2 and -2 when there is array gain. Therefore, if distance of two symbols is drift apart as twice, BER performance is improved about $3 \mathrm{~dB}$. Namely, we are possible to obtain BER performance improvement by applying Alamouti STBC using two receivers because it can obtain the array gain. So, if the Alamouti STBC of MIMO system apply to chaos communication system in fading channel, the deterioration of BER performance is possible to compensate.

\section{IV.CONCLUSION}

MIMO system is able to use multiple antennas to the receiver and transmitter, and to effectively utilize the spatial diversity. In a wireless communication environment, multipath fading is a disorder greatest factor for realizing high-speed data communication. BER performance of chaos communication system is evaluated badly in fading channel. However, the BER performance deterioration can be compensate in Rayleigh channel by applying the Alamouti STBC using the two transmitters and one receiver. Also, if the Alamouti STBC that is used two transmitters and two receivers apply to CDSK system, BER performance is better than existing performance that use only one receiver. Therefore, if the Alamouti STBC of MIMO system apply to chaos communication system in fading channel, the deterioration of BER performance is possible to compensate. And, if you adjust the number of transmitters and receivers according to the channel, effective communication is possible.

\section{ACKNOWLEDGMENT}

This research was supported by Basic Science Research Program through the National Research Foundation of Korea(NRF) funded by the Ministry of Education, Science and Technology. (No. 2012017339)

\section{REFERENCES}

[1] Mikhail Sushchik, Lev S. Tsimring, and Alexander R. Volkovskii; "Performance analysis of correlation-based communication schemes utilizing chaos," IEEE Transactions on, vol.47, no.12, December 2000.

[2] Young Guen Seo, "The world of chaos", Dong-A University, vol.29, pp.520-524, 1989.

[3] Eun Young Jang, J. Wright, "FPGA implementation of chaos-based digital communication system using CPPM", Dept. of Electronic Engineering Graduate School, Dong-A University Busan, Korea, Dec. 2007.

[4] Chen YiPing; Shi Ying; Zhang Dianlun, "Performance of differential chaos-shift-keying digital communication systems over several common channels," Future Computer and Communication (ICFCC), 2010 2nd
International Conference on, vol.2, pp.V2-755,V2-759, 21-24, May 2010 .

[5] Kaddoum, G.; Mai Vu; Gagnon, F., "Performance analysis of differential chaotic shift keying communications in MIMO systems," Circuits and Systems (ISCAS), 2011 IEEE International Symposium on, pp. 1580-1583, 15-18 May 2011.

[6] Chan-Ho Jin; Heung-Gyoon Ryu, "Performance evaluation of chaotic CDSK modulation system with different chaotic maps," ICT Convergence (ICTC), 2012 International Conference on, pp. 603-606, 15-17, Oct. 2012.

[7] Suwa Kim, Junyeong Bok, Heung-Gyoon Ryu, "Performance evaluation of DCSK system with chaotic maps," International Conference On Information Networking (ICOIN), pp. 27-30, Jan. 2013.

[8] Dian-Wu Yue; Zhang, K.Q.T., "Wireless MIMO systems in fading environments: A stabilizing transmission approach," Wireless Communications, Networking and Mobile Computing (WiCOM), 2012 8th International Conference on , pp. 1-8, 21-23, Sept. 2012.

[9] Alamouti, S., "A simple transmit diversity technique for wireless communications," Selected Areas in Communications, IEEE Journal on , vol. 16, no. 8, pp. 1451-1458, Oct 1998.

[10] Jun-Hyun Lee, Heung-Gyoon Ryu, "Anti-jamming performance of chaos communications system using DCSK and CDSK modulation method", The Journal of Korean Institute of Electromagnetic Engineering and Science. vol. 24, no. 4, pp. 417-425, Apr. 2013.

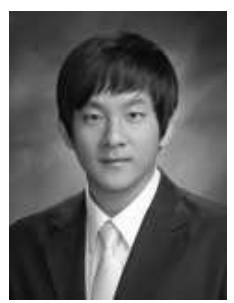

Jun-Hyun Lee was born in Seoul, Republic of Korea in 1989. He received the B.S. degrees in the department of electronic engineering from Chungbuk National University in 2012. Now he is currently working toward M.S. degree at the department of Electronic Engineering, Chungbuk National University, Republic of Korea. His research interests include digital communication system, Secure communication system and wireless communication system.

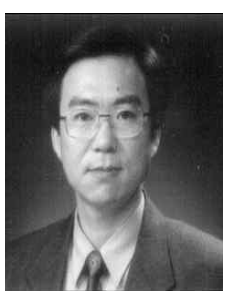

Heung-Gyoon Ryu (M'88) was born in Seoul, Republic of Korea in 1959. He received the B.S. and M.S. and Ph.D. degrees in electronic engineering from Seoul National University in 1982, 1984 and 1989. Since 1988, he has been with Chungbuk National University, Korea, where he is currently Professor of Department of Electrical, Electronic and Computer Engineering in Chungbuk National University. And he worked as Chief of RICIC (research institute of computer, information communication center) in Chungbuk National University from March 2002 to Feb 2004. His main research interests are digital communication systems, communication circuit design, spread spectrum system and communication signal processing. Since 1999, he has worked as reviewer of the IEEE transaction paper. He was a winner of '2002 ACADEMY AWARD' from the Korea Electromagnetic Engineering Society, Korea. He received the "BEST PAPER AWARD" at the 4th International Conference on Wireless Mobile Communications (ICWMC 2008) Athens, Greece, July 27-Aug.1, 2008. Also, He received the "BEST PAPER AWARD" at the International Conference on Advances in Satellite and Space Communications (SPACOMM 2009), Colmar France, July 20-25, 2009. 